\title{
A study of effect of Vitamin D supplementation in Osteoarthritis patients
}

\author{
J. Ashok Vardhan Reddy. ${ }^{1}$, Garikapati S. ${ }^{2}$ \\ ${ }^{1}$ Dr. J. Ashok Vardhan Reddy, Associate Professor, Department of Orthopedics, Maheshwara Medical College \& \\ Hospital, Chitkul, Sangareddy District, Telangana, ${ }^{2}$ Dr. Sridhar Garikapati, Assistant Professor, Department of \\ Orthopedics, Shadan Institute of Medical Sciences \& Research Centre, Peeranchetuvu, Himayathnagar Road, \\ Rangareddy District, Hyderabad, Telangana.
}

Corresponding Author: Dr. Sridhar Garikapati, Assistant Professor, Department of Orthopedics, Shadan Institute of Medical Sciences \& Research Centre, Peeranchetuvu, Himayathnagar Road, Rangareddy District, Hyderabad, Telangana

\begin{abstract}
Introduction: Osteoarthritis (OA) is the common cause of musculoskeletal disability and pain. Osteoarthritis (OA) is a chronic disease characterized by a loss of articular cartilage and changes of the subchondral bone. Lower levels of vitamin D were associated with greater knee pain, poor quadriceps function with poor physical function. Several studies have documented that vitamin D supplementation increases muscle strength, improve physical performance, and decreases risk of falls among older people with low level of serum vitamin D. Materials \&Methods: This present study was conducted at the outpatient clinic of the Department of Orthopedics at Maheswara Medical College \& Hospital, Sangareddy during a February-December 2017 study period.The inclusion criteria were that the participants had symptomatic knee OA and low vitamin D status $(25(\mathrm{OH}) \mathrm{D}<30 \mathrm{ng} / \mathrm{mL})$. Results: Data were analyzed using SPSS Statistics version 22 (SPSS, Inc., Chicago, IL, USA). Comparisonof baseline vs. post-vitamin Dsupplementation data was performed by paired t-test. One-way repeated-measurement ANOVA was used to test the time differences in muscle strength and physical performance. A p-value less than 0.05 for differences and the values were considered to be statistically significant. Dominant grip strength $(\mathrm{p}=0.01)$ and overall physical performance, such as gait speed $(\mathrm{p}<$ 0.001), TUGT ( $<<0.001)$, STS ( $<<0.001)$, and 6MWT $(\mathrm{p}<0.001)$, significantly improved after vitamin. Conclusion: Nevertheless, vitamin D supplementation is a safe and inexpensive way to improve muscle strength and physical function in this population. Based on these findings, we recommend vitamin D supplementation in knee OA patients that have poor physical function.
\end{abstract}

Key words: Osteoarthritis, Vitamin D, articular cartilage, malalignment,

\section{Introduction}

Osteoarthritis (OA) is the common cause of musculoskeletal disability and pain. Osteoarthritis (OA) is a chronic disease characterized by a loss of articular cartilage and changes of the subchondral bone [1]. Knee is one of the most commonly affected joint. Several environmental factors including obesity, malalignment, trauma or joint instability have been associated with knee OA. Other symptoms of disease include joint pain, knee muscle wasting, and decreased range of motion, all of which lead to severe pain and disability in later life [2]. There are many risk factors that lead to early structural changes of the knee among healthy individuals. Vitamin D deficiency may play a role in the

Manuscript Received: $10^{\text {th }}$ June 2018

Reviewed: $20^{\text {th }}$ June 2018

Author Corrected: $27^{\text {th }}$ June 2018

Accepted for Publication: $30^{\text {th }}$ June 2018 pathogenesis of OA. OA coexists frequently with vitamin D deficiency in elderly people. $63 \%$ of primary knee OA patients were found to have low vitamin D status worldwide. Lower levels of vitamin D were associated with greater knee pain, poor quadriceps function with poor physical function. Several studies have documented that vitamin D supplementation increases muscle strength, improves physical performance, and decreases risk of falls among older people with low level of serum vitamin D [3].

Normal bone and cartilage metabolism depend on presence of vitamin D. Vitamin D deficiency has adverse effects on calcium metabolism, osteoblastic activity, matrix ossification, bone density, and articular cartilage turnover. Vitamin D deficiency may lead to 


\section{Original Research Article}

osteoarthritis via reducing the proteoglycan synthesis and increasing the metalloproteinase activity [4]. vitamin D (ergocalciferol) was used in this study for the investigationof the role of vitamin D supplementation on muscle strength and physical performance in knee OApatients with vitamin D insufficiency [5]. Given this disparity in the previous finding regarding vitamin D supplementation in Thailand, vitamin D2 (ergocalciferol) was used in this study for the investigation of the role of vitamin $\mathrm{D}$ supplementation on muscle strength and physical performance in knee OA patients with vitamin D insufficiency and deficiency.

\section{Materials \& Methods}

Place of the study: This present study was conducted at the outpatient clinic of the Department of Orthopedics at Maheswara Medical College \& Hospital, Sangareddy during a February-December 2017 study period.

Type of study: Observational study

Sampling method: Randomly selected

Sample collection: One hundred and thirty-four patients with knee OA agreed to participate in the study.

Inclusion criteria: were that the participants had symptomatic knee $\mathrm{OA}$ and low vitamin $\mathrm{D}$ status $(25(\mathrm{OH}) \mathrm{D}<30 \mathrm{ng} / \mathrm{mL})$. The diagnosis of osteoarthritis is based primarily on patient history, physical examination, and radiographic findings.

Exclusion Criteria: Exclusion criteria included history of knee surgery, primary hyperparathyroidism, rheumatoid or other inflammatory arthritis

Statistical Method: Data were analyzed using SPSS Statistics version 20. Comparison of baseline vs. postvitamin Dsupplementation data was performed by paired t-test. One-way repeated-measurement ANOVA was used to test the time differences in muscle strength and physical performance.

A p-value less than 0.05 for differences and the values were considered to be statistically significant.
Written informed consent was obtained from all participants prior to their participation in the study. The Endocrine Society guidelines suggest that 50,000 IU of vitamin D 2 taken once a week for eight weeks is necessary to achieve the levels of serum 25(OH)D consistently above $30 \mathrm{ng} / \mathrm{mL}$ in adults. All participants were evaluated for knee pain using WOMAC and VAS evaluation instruments. VASscore is based on a $0-10$ point scale, with a higher score indicating a higher level of pain. The participants were asked to put a mark on the line indicating their pain intensity at the present time in response to the following question: "If " 0 " is "no pain" and " $10 "$ is "the worst pain", where is your average pain intensity now on the visual analog score (VAS). Total WOMAC score represented the sum of three subscales, including pain, stiffness, and physical function. A higher WOMAC score indicates worse pain, more stiffness, and increased functional limitations.

At baseline of six months, muscle strength and physical performance were measured by physical therapists. Grip strength was assessed by grip strength dynamometer (Takei Scientific Instruments Co. Ltd., Tokyo, Japan) (kilograms). Knee extension force was measured by a handheld Micro FET 2 dynamometer (Hoggan Scientific LLC, Salt Lake City, UT, USA) (Newtons). The participant sat on the treatment table with knees flexed 90and the dynamometer was applied to the anterior part of the leg, $5 \mathrm{~cm}$ above the transmalleolar axis and perpendicular to the tibial crest. The participant raised their lower legs up and held against a maximum persistent force position ( $5 \mathrm{~s}$ ) applied by a physical therapist. Four tests were used to evaluate physical performance. The first test was the 4-m gait speed test, which measures the time needed to walk four meters, calculated as meters per second [3]. The second test was the Timed Up and Go Test (TUGT), which measured the time needed to stand up from a chair, walk three meters, and return to the chair and sit down (seconds) ${ }^{4}$. The third test was the five times sit-to-stand test (STS), which recorded the time needed to perform five repeated chair stands without the use of arms (seconds) ${ }^{5}$. The last of the four tests was the six-minute walk test (6MWT), which measured the distance a patient could walk in six minutes (in meters).

\section{Results}

Data were analyzed using SPSS Statistics version 20. Comparison of baseline vs. post-vitamin Dsupplementation data was performed by paired t-test. One-wayrepeated-measurement ANOVA was used to test the time differences in muscle strength and physicalperformance. A p-value less than 0.05 for differences and the values were considered to be statistically significant.WOMAC scores did not change significantly between baseline and six months. However, VAS decreased significantly after treatment $(p=0.004)$ and the PCS of SF-12 improved significantly after supplementation treatment $(\mathrm{p}=0.005)$. 
Original Research Article

Table-1: Effect of Vitamin D supplementation on Muscle Strength and Physical Performance.

\begin{tabular}{|c|l|c|c|c|}
\hline S No & parameters & baseline & 6months & p-value \\
\hline 1 & Grip strength (kg) & & & \\
& Dominant (kg) & $21.15 \pm 0.10$ & $22.30 \pm 0.21$ & 0.01 \\
& Non dominant (Kg) & $19.17 \pm 0.21$ & $19.39 \pm 0.40$ & 0.13 \\
\hline 2 & Knee extension force & $351.02 \pm 5.02$ & $353.43 \pm 5.32$ & 0.30 \\
& Symptomatic leg (N) & $367.12 \pm 5.34$ & $369.51 \pm 5.45$ & 0.04 \\
\hline 3 & Non- symptomatic leg (N) & & & $<0.001$ \\
& Physical Performance & $0.87 \pm 0.12$ & $1.10 \pm 0.03$ & $<0.001$ \\
& Gait speed (m/s) & $8.82 \pm 0.12$ & $7.65 \pm 0.19$ & $<0.001$ \\
& TUGT (S) & $14.24 \pm 0.89$ & $13.21 \pm 0.76$ & $<0.001$ \\
\hline
\end{tabular}

Effects on Muscle Strength and Physical Performance Dominant grip strength $(\mathrm{p}=0.01)$ and overall physical performance, such as gait speed ( $p<0.001)$, TUGT $(\mathrm{p}<0.001)$, STS $(\mathrm{p}<0.001)$, and 6 MWT ( $<0.001)$, significantly improved after vitamin D supplementation, but there were no significant difference observed for non-dominant grip strength and knee extension force between baseline and post-treatment $(\mathrm{p}>0.05)$ are presented (Table 1)

\section{Discussion}

In the above study, regarding muscle strength and physical performance, we found that knee OA patients significantly improved grip strength and physical performance, but did not improve knee extension force. In this aspect, our results are consistent with the findings of several previous studies. Zhu et al. reported that hip muscle strength and TUGT improved significantly after 1000 IU/day vitamin D2 supplementation for one year in older women with vitamin D insufficiency [6]. Lagari et al. reported that vitamin D supplementation might be most beneficial in older populations with poor physical function [7].

Sato et al. found that the mean of type II muscle fiber diameter and percentage of type II fibers increased significantly after $1000 \mathrm{IU} /$ day vitamin D2 treatment over two years in elderly patients with post-stroke hemiplegia [8]. Ceglia et al. reported that intra myonuclear VDR concentration increased 30\% and total (type I and II) muscle fiber size increased $10 \%$ after vitamin D. supplementation in mobility-limited elderly women [9]. However, some studies have reported that vitamin D supplementation did not improve muscle strength or physical function. Kenny et al. found that vitamin D supplementation did not improve muscle strength or physical performance in a group of healthy community-dwelling older men [10]. These conflicting findings may be attributed to differences in populations, disease advancement, or measurements applied, or to incomplete control of confounding variables. None theless, conclusions should be drawn with caution on whether the characteristics of studied participants or the dose of vitamin D used are of significance, as these studies were heterogeneous with regards to most aspects. Various outcome measures have been documented by different investigators and even in the case of measurements of similar characteristics, different methods have been applied, making it difficult to compare studies directly. A strength of this study is the finding that a high dose and a long-term intervention of vitamin D2 supplementation was effective in raising $25(\mathrm{OH}) \mathrm{D}$ concentrations. It is possible that achieved serum $25(\mathrm{OH}) \mathrm{D}$ levels may improve muscle function by increasing muscle strength and physical performance in knee OA patients. Higher serum 25(OH)D concentrations may be essential in skeletal muscle, particularly for the elderly with limited mobility. On the other hand, increasing $25(\mathrm{OH}) \mathrm{D}$ levels in healthy populations do not relate to any improvement of muscle function.

Therefore, patients with impaired mobility may be more sensitive to the improvement in physical functioning by vitamin D supplementation. Previous studies indicated that vitamin D supplementation in the elderly with vitamin D insufficiency reduced an atrophy of type II muscle fiber and increased the size of type I and II muscle fiber, as well as VDR concentration. Actually, knee OA patients with poor muscle function and vitamin $\mathrm{D}$ deficiency may be the most likely to benefit from vitamin D supplementation. This study has several mentionable limitations. First, the controlled beforeafter design of this study did not include a control group. The lack of randomization, and our decision not to evaluate the sensitivity of drug effect, potentially weaken our findings relative to the therapeutic effect of vitamin D supplementation. Second, the sample size 
was small and the proportion of men was low, both of which prevented us from establishing the clinical relevance, particularly regarding changes in muscle strength.Third, we assayed markers of oxidative damage using plasma protein carbonyls that were not directly measured in skeletal muscle. Finally, $8.37 \%$ of patients were lost to follow-up. While this rate is higher than can be considered ideal, the loss to follow-up rate in the present study was lower than loss to follow-up rates reported from other studies.

\section{Conclusions}

In conclusion, our results suggest that 40,000 IU of vitamin D supplementation reduced oxidative protein damage, improved quality of life, and improved grip strength and physical performance. Accordingly, vitamin D treatment decreases current pain using VAS, but does not reduce pain during physical activity, as determined by WOMAC score.Vitamin D supplementation is a safe and inexpensive way to improve muscle strength and physical function in our population. Based on these findings, we can strongly recommend vitamin $\mathrm{D}$ supplementation in knee OA patients that have poor physical function.

\section{Contribution by different authors during the study} process- The principal author involved in Conception or design of the work, Data collection, Data analysis and interpretation and co author involved in Drafting the articleCritical revision of the article.

Study adds to the existing knowledge- Vitamin D supplementation leads to reduced pain, stiffness or functional loss over a 3 -year period. On the basis of these findings we consider that vitamin D supplementation has no role in the management of knee OA.

Conflict of interest: None declared.

Funding: Nil, Permission from IRB: Yes

\section{References}

1. Castañeda S, Roman-Blas JA, Largo R, HerreroBeaumont G. Subchondral bone as a key target for osteoarthritis treatment. DOI:10.1016/j.bcp.2011.09.018

\section{Original Research Article}

2. Bisch off HA, Stähelin HB, Dick W et al. Effects of vitamin $\mathrm{D}$ and calcium supplementation on falls: a randomized controlled trial. DOI:10.1359/jbmr. 2003. 18.2.343

3. Cruz-Jentoft AJ, Baeyens JP, Bauer JM, Boirie Y, et al. Sarcopenia: European consensus on definition and diagnosis: Report of the European Working Group on Sarcopenia in Older People. DOI:10.1093/ ageing/ afq034

4. Maly MR, Costigan PA, Olney SJ. Determinants of self-report outcome measures in people with knee osteoarthritis. DOI:10.1016/j.apmr.2005.08.110

5.Mijnarends DM, Meijers JM, Halfens RJ et al. Validity and reliability of tools to measure muscle mass, strength, and physical performance in communitydwelling older people: a systematic review. DOI:10. 1016/j. jamda.2012.10.009

6. Zhu K, Austin N, Devine Aet al. A randomized controlled trial of the effects of vitamin D on muscle strength and mobility in olderwomen with vitamin D insufficiency. DOI:10.1111/j.1532-5415.2010. 03142.x

7. Lagari V, Gómez-Marín O, Levis S. The role of vitamin $\mathrm{D}$ in improving physical performance in the elderly. DOI:10.1002/jbmr.1949

8. Sato Y, Iwamoto J, Kanoko T, Satoh K. Low-dose vitamin $\mathrm{D}$ prevents muscular atrophy and reduces falls and hip fractures in womenafter stroke: a randomized controlled trial. DOI:10.1159/000087203

9. Ceglia L, Niramitmahapanya S, da Silva MoraisMet al. A randomized study on the effect of vitamin $\mathrm{D}_{3}$ supplementation on skeletal muscle morphology and vitamin D receptor concentration in older women. DOI:10.1210/jc.2013-2820

10. Kenny AM, Biskup B, Robbins B et al. Effects of vitamin D supplementation on strength, physical function, and health perception in older, communitydwelling men. J Am Geriatr Soc. 2003 Dec;51 (12): 1762-7.

\section{How to cite this article?}

J. Ashok Vardhan Reddy, Garikapati S. A study of effect of Vitamin D supplementation in Osteoarthritis patients. Surgical Update: Int J surg Orthopedics. 2018;4(2):90-93. doi:10.17511/ijoso.2018.i02.06. 